\title{
Hyper-Aging Dynamics of Nanoclay Suspension
}

\author{
A. Shahin and Yogesh M Joshi* \\ Department of Chemical Engineering, Indian Institute of Technology Kanpur, Kanpur 208016, India
}

\section{Supporting Information}

ABSTRACT: Aqueous suspension of nanoclay Laponite undergoes structural evolution as a function of time, which enhances its elasticity and relaxation time. In this work, we employ an effective time approach to investigate long-term relaxation dynamics by carrying out creep experiments. Typically, we observe that the monotonic evolution of elastic modulus shifts to lower aging times, while maxima in viscous moduli get progressively broader for experiments carried out on a later date after preparation (idle time) of the nanoclay suspension. Application of effective time theory produces a superposition of all the creep curves irrespective of their initial state. The resulting dependence of the relaxation time on aging time shows very strong hyper-aging dynamics at short idle times, which progressively weakens to demonstrate a linear dependence in the limit of very long idle times. Remarkably, this behavior of nanoclay suspensions is akin to that observed for polymeric glasses. Consideration of aging as a first-order process suggests that continued hyper-aging

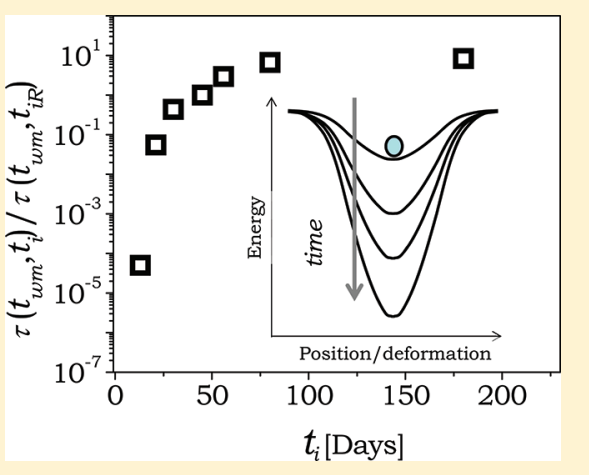
dynamics causes cessation of aging. The dependence of relaxation time on aging time, therefore, must attenuate eventually producing linear or weaker dependence on time in order to approach a progressively low-energy state in the limit of very long times as observed experimentally. We also develop a simple scaling model based on a concept of aging of an energy well, which qualitatively captures various experimental observations very well, leading to profound insight into the hyper-aging dynamics of nanoclay suspensions.

\section{INTRODUCTION}

Clays are ubiquitous in nature and industry. Particularly, 2:1 smectite clays find applications as rheology modifiers in health care, personal care, petroleum, and paint industries due to their nanometric size, anisotropic (sheet-like) shape, and affordable cost. Common examples of such clay minerals are Montmorrillonite, Bentonite, and Laponite, which have layer thickness of around $1 \mathrm{~nm}$ with aspect ratio in the range 25$1000 .^{1,2}$ These clay minerals are highly hydrophilic and swell in water. Typically, upon addition of clay to water, the suspension slowly transforms itself from a flowing liquid to a highly viscous soft solid (that supports its weight), suggesting the presence of jamming. ${ }^{3-8}$ Such a transformation is also usually accompanied by evolution of its structure and various physical properties as a function of time. ${ }^{9,10}$ In this work, we employ an effective time framework to study the jamming transition and relaxation dynamics of an aqueous Laponite suspension using rheological tools. We also estimate the relaxation time dependence on the time elapsed since preparation, which shows a striking similarity to that of molecular glasses.

Synthetic hectorite clay, Laponite, has the chemical formula $\left(\mathrm{Na}_{+0.7}\left[\left(\mathrm{Si}_{8} \mathrm{Mg}_{5.5} \mathrm{Li}_{0.3}\right) \mathrm{O}_{20}(\mathrm{OH})_{4}\right]_{-0.7}\right){ }^{11}$ Laponite has a disklike shape with thickness $1 \mathrm{~nm}$ and diameter $25-30 \mathrm{~nm}$ and is farely monodisperse. ${ }^{11,12}$ The unit crystal of Laponite is composed of an octahedral magnesia sheet sandwiched between two tetrahedral silica sheets. Isomorphic substitution of divalent magnesium by monovalent lithium creats a paucity of positive charge within the layer, which is compensated by positive sodium ions present on the face of the Laponite particle. On the edge of Laponite particles, where the crystal structure is broken, the presence of amphoteric groups renders the same positive charge at low $\mathrm{pH}$ but a negative charge at high $\mathrm{pH} .{ }^{13}$ At $\mathrm{pH}$ 10, Laponite particles are believed to have a positive edge charge. ${ }^{14}$ In an aqueous medium, sodium ions dissociate, giving the face of Laponite a permanant negative charge. In an aqueous Laponite suspension, the competition between repulsion originating from negatively charged faces and attraction between a negatively charged face and positively charged edge controls the phase behavior. ${ }^{15,16}$ In the concentrated Laponite suspension (typically beyond 2 wt \%), based on spectroscopic studies, some groups propose the microstructure to be dominated by repulsion, leading to a repulsive or Wigner glass. ${ }^{17-19}$ Laponite particles do not physically touch each other in a Wigner glass and remain selfsuspended in the aqueous media due to repulsion. The other school claims the phase behavior of a Laponite suspension to be dominated by attractive interactions among Laponite particles leading to a gel. ${ }^{20-22}$ At intermediate Laponite concnetrations ( 1.1 to $2.4 \mathrm{wt} \%$ ), one of the proposals suggests that the system can evolve as either a glass or a gel depending upon the state of the system owing to its complex energy landscape. ${ }^{23}$ In addition to these claims, aqueous suspensions of Laponite often demonstrate an anisotropic phase, ${ }^{24-26}$ which

Received: December 30, 2011

Revised: March 9, 2012

Published: March 13, 2012 
recently has been reported to have a pronounced presence near the air interface. ${ }^{13}$

Macroscopically, the addition of Laponite to water leads to an increase in viscosity as a function of elapsed time so that apparently the flowing liquid gets converted to a soft, pasty material over a duration ranging from minutes to hours depending on the concentration of Laponite., ${ }^{9,27}$ Depending upon the microstructure of the suspension, whether a repulsive glass or an attractive gel, such a transformation can be represented as either a glass transition or a gelation. Without going into complications of terminology, we address this transformation by a generic term: jamming transition. Beyond the jamming transition, the viscosity and elastic modulus of the Laponite suspension continue to increase as a function of time, a phenomenon usually known as physical aging. ${ }^{28}$ Rheologically, such transformations are analyzed by studying the evolution of $G^{\prime}$ and $G^{\prime \prime}$. For experiments performed on comparatively young samples, subsequent to shear melting, $G^{\prime \prime}$ is higher than $G^{\prime}$ and the material exhibits a liquid-like response. Eventually, $G^{\prime}$ crosses over $G^{\prime \prime},{ }^{15,27}$ and the corresponding time marks the jamming transition. ${ }^{29,30}$ During the aging process, the relaxation time of the material also shows an enhancement as a function of time. Typically, if the dependence of relaxation time on age is weaker than linear, the phenomenon is characterized as subaging, ${ }^{31,32}$ while if the same is stronger than linear, the phenomenon is addressed as hyperaging. ${ }^{33,34}$ The application of a strong deformation field in a rheological experiment reverses the aging by causing "shear induced" melting of the soft solid, producing a liquid. However, in the case of a Laponite suspension, the structural evolution is observed not to be completely reversible over very long durations of the time since sample preparation (time scales of days). ${ }^{15}$ Typically, shear melting of progressively older samples is observed to produce a liquid with greater viscosity.

An aqueous Laponite suspension, by virtue of its timedependent physical behavior, does not follow time-translational invariance. ${ }^{35,36}$ Such behavior, therefore, does not let the material obey linear viscoelastic principles. Recently, our group proposed a methodology based on an effective time theory and showed that linear viscoelastic principles can be successfully applied to such time-dependent materials. ${ }^{10,35,37}$ The application of an effective time approach directly leads to estimation of the dependence of relaxation time on the waiting time. In this work, we use the effective time theory to study the evolution of an aqueous suspension of Laponite using rheological tools that gives new insights into the long-term evolution of a soft glassy material.

\section{MATERIALS AND EXPERIMENTAL PROTOCOL}

In this work, we have used an aqueous suspension of $2.8 \mathrm{wt} \%$ Laponite RD (Southern Clay Products Inc.) having $0.5 \mathrm{wt} \%$ poly(ethylene oxide) (Loba Chemie, mol wt 6000). Oven-dried white powders of Laponite $\mathrm{RD}$ and $\mathrm{PEO}$ were added simultaneously to deionized water with $\mathrm{pH} 10$ (maintained by addition of $\mathrm{NaOH}$ ) and mixed using an Ultra Turrex Drive for a period of $45 \mathrm{~min}$. The resulting suspension was stored in airtight polypropylene bottles at 30 ${ }^{\circ} \mathrm{C}$. We denote the period of storage as idle time $\left(t_{\mathrm{i}}\right)$ of the system, usually denoted in days. In this work, we carry out creep experiments using a stress-controlled rheometer, AR 1000. For a given idle time, the suspension was loaded in a concentric cylindrical geometry with an inner diameter of $28 \mathrm{~mm}$ and a gap of $1 \mathrm{~mm}$. To ensure a uniform initial state for a given idle time, the suspension was shear-melted by applying oscillatory shear having a stress magnitude of $80 \mathrm{~Pa}$ at $0.1 \mathrm{~Hz}$ frequency for $20 \mathrm{~min}$. Subsequently, in an aging step, the evolution of elastic and viscous moduli of the suspension was monitored by applying small-amplitude oscillatory shear with stress magnitude of 1 $\mathrm{Pa}$ and frequency of $0.1 \mathrm{~Hz}$. Aging experiments were carried out for a predetermined time, known as aging time, following which creep experiments were carried out by applying a constant stress having a magnitude of $5 \mathrm{~Pa}$. In order to avoid drying, the free surface of the sample was covered with a thin layer of low-viscosity silicon oil. All the experiments were carried out at $25^{\circ} \mathrm{C}$.

It should be noted that the addition of poly(ethylene oxide) to aqueous Laponite suspensions can alter the aging dynamics of the suspension, and hence, the aging behavior of Laponite-PEO suspensions could be different compared to that of pure Laponite suspensions. The addition of low-molecular-weight PEO slows down the aging dynamics of suspensions due to steric hindrance, ${ }^{38}$ whereas the addition of high-molecular-weight PEO forms shake gels, as the PEO chains have the ability to form bridges between Laponite platelets. $^{38,39}$

\section{RESULTS AND DISCUSSION}

In Figure 1, the evolution of elastic and viscous moduli of shear-melted Laponite suspensions is plotted as a function of

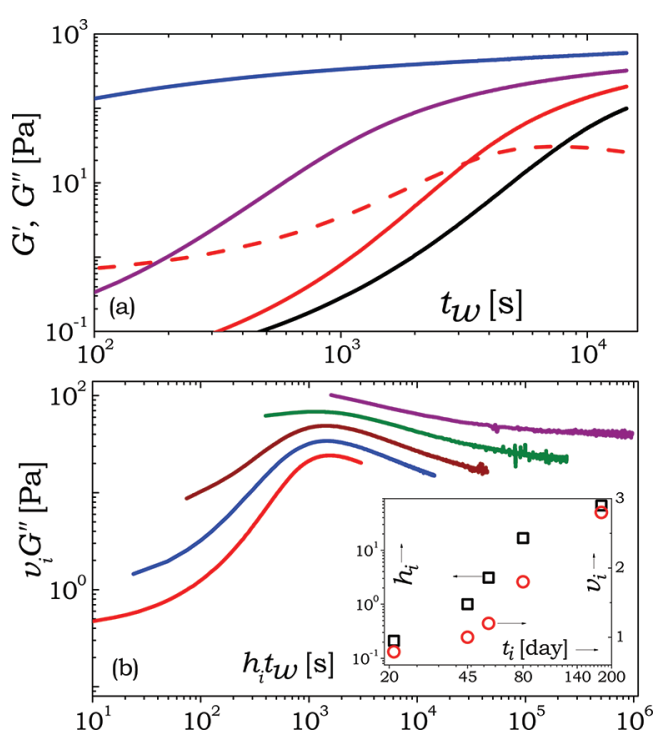

Figure 1. Evolution of elastic modulus and viscous modulus. (a) Elastic modulus (solid line) is plotted as a function of aging time for experiments carried out on various numbers of days elapsed after preparation of the Laponite suspension (idle time, $t_{\mathrm{i}}$ ). From right to left: black line, $t_{\mathrm{i}}=13$ days; red line, 21 days; purple line, 45 days; blue line, 80 days. We have also plotted $G^{\prime \prime}$ for 21 day data as a dashed red line. (b) Viscous modulus is plotted as a function of aging time (from top to bottom: 180, 80, 56, 45, 21 days). The curves are shifted vertically and horizontally for clarity. It can be seen that, with an increase in idle time, the maximum in $G^{\prime \prime}$ becomes broad. Inset shows the horizontal shift factors (black squares) and vertical shift factors (red circles) used to demarcate the curvature of $G^{\prime \prime}$.

time, for experiments carried out on various days after preparation of suspension (idle time, $t_{\mathrm{i}}$ ). It can be seen that the evolution of $G^{\prime}$, which is monotonic and has self-similar curvature, shifts to smaller times for experiments carried on a later date after preparation (idle time). $G^{\prime \prime}$, on the other hand, demonstrates a maximum whose breadth becomes progressively broader at later idle times. We carry out such aging experiments at various idle times for predetermined aging times $\left(t_{\mathrm{w}}\right)$ and apply constant stress. The consequent creep curves obtained on a 21-day-old Laponite suspension at various $t_{\mathrm{w}}$ values are plotted in the inset of Figure 2. It can be seen that 


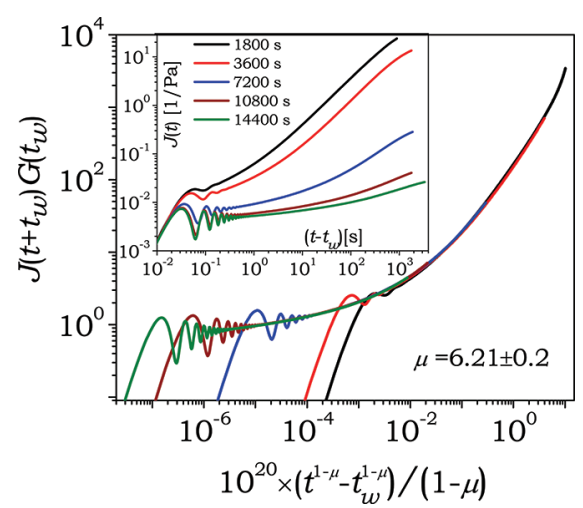

Figure 2. Time-aging time superposition for creep curves generated on $t_{\mathrm{i}}=21$ days. The inset shows creep curves obtained at various waiting times (from top to bottom: 1800, 3600, 7200, 10800 , and 14 $400 \mathrm{~s})$.

lesser compliance is induced for experiments carried out at higher $t_{\mathrm{w}}$. The aging-time-dependent creep flow behavior implies an inapplicability of the time-translational invariance (TTI). Therefore, the Boltzmann superposition principle is not applicable to the system. This principle is mathematically represented as ${ }^{40}$

$$
\gamma(t)=\int_{-\infty}^{t} J\left(t-t_{\mathrm{w}}\right) \frac{\mathrm{d} \sigma}{\mathrm{d} t_{\mathrm{w}}} \mathrm{d} t_{\mathrm{w}}
$$

where $\gamma$ is the strain in the material at the present time $t$ under the application of stress $\sigma$ applied at time $t_{\mathrm{w}} . J$ is compliance, which solely depends on $t-t_{\mathrm{w}}$. However, as shown in the inset of Figure 2, for an aqueous suspension of Laponite, $J$ shows an additional dependence on $t_{\mathrm{w}}$ leading to $J=J\left(t-t_{\mathrm{w}}, t_{\mathrm{w}}\right)$ invalidating the Boltzmann superposition principle. This behavior is normally observed when the relaxation time increases as a function of aging time. Under such circumstances, it is customary to replace real time by an effective time obtained by rescaling the time-dependent relaxation process with a constant relaxation time. ${ }^{36}$ The effective time is defined as ${ }^{35,36}$

$$
\xi(t)=\int_{0}^{t} \tau_{0} \mathrm{~d} t^{\prime} / \tau\left(t^{\prime}\right)
$$

where $\tau$ is relaxation time, while $\tau_{0}$ is constant relaxation time associated with the effective time. In the effective time domain, since the relaxation time remains constant, eq 1 can be applied to the present system by replacing $\left(t-t_{\mathrm{w}}\right)$ by $\left[\xi(t)-\xi\left(t_{\mathrm{w}}\right)\right]$ in the expression for compliance: $J\left(t-t_{\mathrm{w}}\right)=J\left[\xi(t)-\xi\left(t_{\mathrm{w}}\right)\right]$. However, in order to estimate effective time, according to eq 2, it is necessary to know the dependence of the relaxation time on aging time: $\tau\left(t^{\prime}\right)$. It is usually observed that an aqueous suspension of Laponite, when it has a soft solid-like consistency, follows a power law dependence of the relaxation time on aging time given by $\tau=A \tau_{\mathrm{m}}^{1-\mu} t^{\prime \mu},{ }^{28,35,36}$ where $\tau_{\mathrm{m}}$ is the microscopic time scale associated with the suspension (discussed below) and $A$ is a constant prefactor, while $\mu$ is the logarithmic rate of change of the relaxation time with respect to aging time. When the Laponite suspension is in the liquid state $\left(G^{\prime}<G^{\prime \prime}\right)$, it is observed to follow an exponential dependence on time given by $\tau\left(t^{\prime}\right)=\tau_{\mathrm{m}} \exp \left(\alpha t^{\prime}\right) .{ }^{35}$ [Aging dynamics of freshly prepared Laponite suspensions studied using dynamic light scattering experiments also show initial exponential growth followed by a linear growth of relaxation time. ${ }^{41-43}$ However, we believe that this behavior is related to cage formation dynamics ${ }^{5}$ and is different compared to that discussed in the present work wherein experiments are carried out on at least 13-day-old shear-melted Laponite suspension]. In Figure 2, we have plotted creep curves associated with a 21day-old Laponite suspension over the duration of aging times in the range 1800-14 $400 \mathrm{~s}$. Similar data for day 13 and day 30 are reported in ref 35. As shown in Figure 1, over this duration, the elastic modulus is greater than the viscous modulus, suggesting the sample to be in a solid-like state. For power law dependence, the effective time elapsed since application of a deformation field according to eq 2 is given by

$$
\xi(t)-\xi\left(t_{\mathrm{w}}\right)=\tau_{0} \tau_{\mathrm{m}}^{\mu-1}\left(t^{1-\mu}-t_{\mathrm{w}}^{1-\mu}\right) /[A(1-\mu)]
$$

If we assume $\tau_{0}=\tau\left(t_{\mathrm{wm}}\right)$, where $t_{\mathrm{wm}}$ is maximum aging time (14400 s) employed in the experiments, we get

$$
\xi(t)-\xi\left(t_{\mathrm{w}}\right)=t_{\mathrm{wm}}^{\mu}\left(t^{1-\mu}-t_{\mathrm{w}}^{1-\mu}\right) /(1-\mu)
$$

It can be seen that creep compliance demonstrates an excellent superposition for a certain unique value of $\mu$, when plotted against $\left(t^{1-\mu}-t_{\mathrm{w}}^{1-\mu}\right) /(1-\mu)$ as shown in Figure 2 . The unique value of $\mu$ necessary to obtain superposition suggests a dependence of the relaxation time on aging time given by $\mu$ $=\mathrm{d} \ln \tau / \mathrm{d} \ln t_{\mathrm{w}}$. Figure 2, therefore, successfully validates the Boltzmann superposition principle in the effective time domain. Importantly, for soft glassy materials including nanoclay suspensions, the Boltzmann superposition principle in an effective time domain not only gets validated for experiments performed at different aging times, but also is observed to get validated for different stress histories and different temperature histories as discussed in the literature. ${ }^{10,35,37}$ It should be noted that the procedure for the estimation of $\mu$ using the effective time theory is different from the conventional procedure. $^{28,44-46}$ In the conventional technique, which is from Struik, ${ }^{28}$ the creep data are considered over only $10 \%$ of aging time in order to avoid the effect of aging during the course of creep flow. The value of $\mu$ is obtained from a superposition by horizontally shifting the creep data. The effective time procedure, on the other hand, allows the use of creep data in its entirety to produce a superposition for a unique value of $\mu$.

We carried out the creep experiments as shown in the inset of Figure 2 at various idle times in the range 13-180 days. Similar to that shown in Figure 2, the creep curves associated with various idle times also demonstrate superposition when plotted against $\left(\left[t^{1-\mu}-t_{\mathrm{w}}^{1-\mu}\right] /(1-\mu)\right)$, which we have described in Figure 3. Unlike all the explored idle times, experiments carried out on $t_{\mathrm{i}}=13$ days demonstrate exponential dependence of relaxation time on aging time: $\tau\left(t^{\prime}\right)=\tau_{\mathrm{m}} \exp \left(\alpha t^{\prime}\right)$. This is because the sample on day 13 is observed to be in the liquid state $\left(G^{\prime}<G^{\prime \prime}\right)$ over most of the explored aging times. According to eq 2, effective time elapsed since the application of deformation for exponential dependence is given by $\xi(t)-\xi\left(t_{\mathrm{w}}\right)=\left(\tau_{0} / \tau_{\mathrm{m}}\right)\left[\exp \left(-\alpha t_{\mathrm{w}}\right)-\right.$ $\exp (-\alpha t)] / \alpha$. If we assume $\tau_{0}=\tau\left(t_{\mathrm{wm}}\right)$,

$$
\xi(t)-\xi\left(t_{\mathrm{w}}\right)=\left[\exp \left(\alpha t_{\mathrm{wm}}\right)\right]\left[\exp \left(-\alpha t_{\mathrm{w}}\right)-\exp (-\alpha t)\right] / \alpha
$$

We have plotted normalized compliance against $\left[\exp \left(-\alpha t_{\mathrm{w}}\right)\right.$ $-\exp (-\alpha t)] / \alpha$ in Figure 3a for day 13 creep data. For all the other explored idle times ( 21 days and beyond), samples show power law dependence. The creep curves on various idle times 

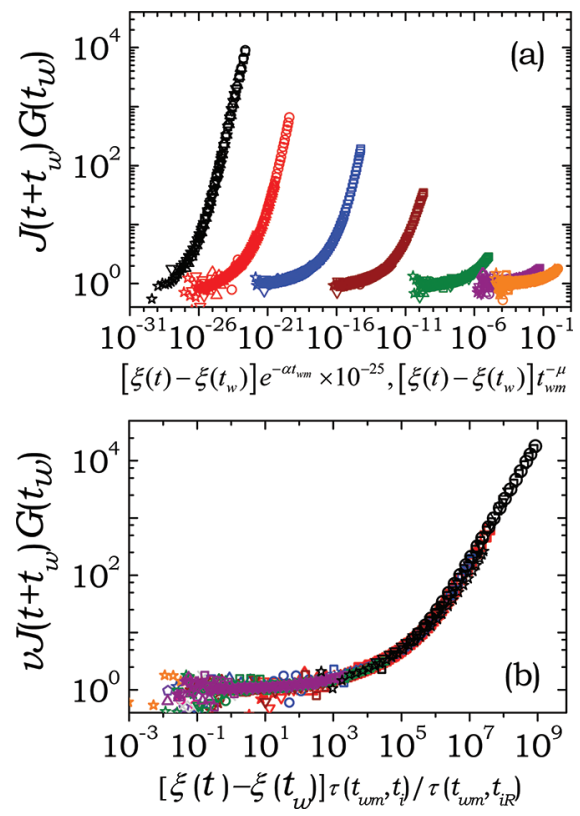

Figure 3. (a) Time-aging time superpositions obtained using the effective time approach on various idle times (from left to right: 13,21, $30,45,56,80,180$ days). (b) Comprehensive superposition, wherein all the creep curves are horizontally shifted onto a creep curve of 45 days. Vertical shift factor $v$ in (b) is used to carry out vertical adjustment and is always in the range 1-1.2.

demonstrate a superposition as shown in Figure 3a for unique values of $\mu$ that depend only on $t_{\mathrm{w}}$. In Figure 4 , we plot $\mu$ as a

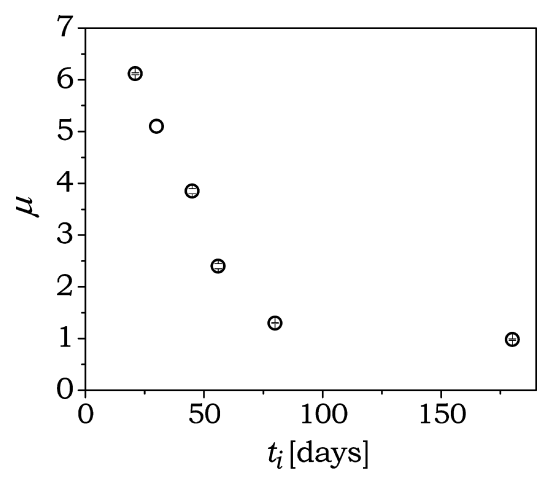

Figure 4. Parameter $\mu$ necessary to obtain the superposition shown in Figure 3 a plotted as a function of idle time.

function of idle time. It can be seen that, in the initial idle time region, $\mu$ is significantly greater than unity, suggesting hyperaging dynamics. With an increase in idle time, $\mu$ decreases rapidly and in the limit of high idle times reaches a plateau around unity, suggesting a linear dependence of the relaxation time on aging time.

All the superpositions plotted in Figure 3a have self-similar curvatures. The superpositions can therefore be shifted horizontally to obtain a comprehensive superposition as shown in Figure $3 \mathrm{~b}$. In Figure 3a, the individual superposition at each idle time is plotted as a function of $\left[\xi(t)-\xi\left(t_{\mathrm{w}}\right)\right] t_{\mathrm{wm}}^{-\mu}$ (for day 13 data, the superposition is plotted against $[\xi(t)-$ $\left.\left.\xi\left(t_{\mathrm{w}}\right)\right] \exp \left(-\alpha t_{\mathrm{wm}}\right)\right)$. The constant relaxation time $\left(\tau_{0}\right)$ associated with each superposition is the relaxation time associated with the maximum aging time at that idle time: $\tau_{0}=$ $\tau\left(t_{\mathrm{wm}}, t_{\mathrm{i}}\right)$. Therefore, if the comprehensive superposition does exist, then the horizontal shift factor is equal to $\tau\left(t_{\mathrm{wm}} / t_{\mathrm{i}}\right) / \tau\left(t_{\mathrm{wm}} /\right.$ $\left.t_{\mathrm{iR}}\right)$, where $t_{\mathrm{iR}}$ is the reference idle time. In Figure 5, we plot

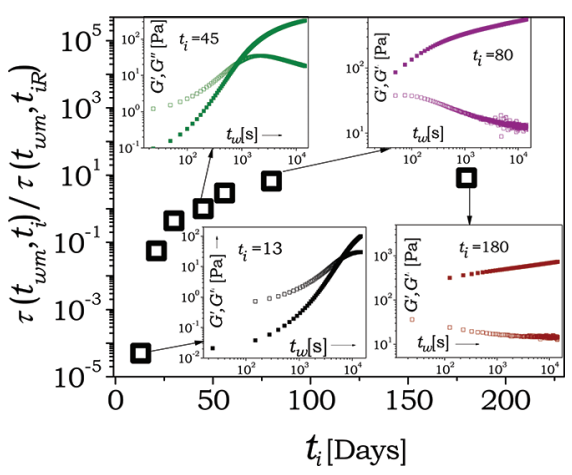

Figure 5. Ratio of relaxation time at $t_{\mathrm{wm}}=14400 \mathrm{~s}$ on respective idle times to that at the reference idle time plotted as a function of idle time. The various insets represent the evolution of elastic and viscous moduli at respective idle times.

$\tau\left(t_{\mathrm{wm}} / t_{\mathrm{i}}\right) / \tau\left(t_{\mathrm{wm}} / t_{\mathrm{iR}}\right)$ as a function of idle time $\left(t_{\mathrm{iR}}=45\right.$ days $)$. We also plot evolutions of elastic and viscous moduli associated with a few idle times as insets on the same plot. Overall, the relaxation time increases with an increase in idle time; however, the maximum change is observed between points associated with 13 day and 21 day data. On day 13 , the sample was in a liquid state $\left(G^{\prime}<G^{\prime \prime}\right)$ at those aging times when creep experiments were carried out. On the other hand, on day 21 the sample was in a solid state $\left(G^{\prime}>G^{\prime \prime}\right)$ when creep experiments were carried out. The jump in relaxation time of over three decades between days 13 and 21, therefore, suggests a liquidsolid jamming transition in an aqueous suspension of Laponite. At greater idle times, when the system is always in the solid state (when creep experiments were carried out), the relaxation time shows a comparatively weaker enhancement.

It is important to note that the hyper-aging behavior observed in the present work should not be confused with the hyper-diffusion reported for an aqueous Laponite suspension. For example, on one hand Bandyopadhyay and co-workers ${ }^{34}$ observed the dependence of the relaxation time on aging time to be stronger than linear $(\mu=1.8)$; on the other hand, they reported faster than exponential relaxation at the explored aging times. The former phenomenon is represented as hyper-aging, while the latter is termed hyper-diffusion. In the literature, hyper-diffusive behavior has been observed for Laponite suspensions even though hyper-aging is absent. ${ }^{42,43}$ We therefore believe that hyper-aging and hyper-diffusion phenomena are unrelated with the latter caused by ballistic movement associated with elastic deformation originating from heterogeneous local stress. ${ }^{47}$ In the case of glassy materials, scaling arguments suggest relaxation time scales as aging time, as this is the only time scale available to the system. The very fact that glassy material shows a stronger than linear relationship suggests that processes other than simple physical aging with different time scales are influencing the dynamics. We therefore believe that irreversible dynamics shown by aqueous Laponite suspensions might be related to observed hyper-aging behavior. However, we feel that further work is necessary in order to understand the physical origin.

The time dependency associated with aqueous suspensions of Laponite is related to the thermodynamically out-ofequilibrium character of the same. Such time-dependent 
behavior, typically addressed as physical aging, is also observed in many out-of-equilibrium systems such as soft glassy materials, ${ }^{35,48}$ spin glasses, ${ }^{31,49}$ and polymeric glasses. ${ }^{28,58,51} \mathrm{~A}$ simple observation that the addition of around $1.1 \mathrm{vol} \%$ of Laponite discs $(\sim 2.8$ wt \%) enhances the viscosity of water by over 5 orders of magnitude suggests that translational diffusivity of the nanometric particles is significantly hindered. Owing to such reduced mobility, only the limited part of the phase space is accessible to the system. Nonetheless, highly constrained motion of the jammed Laponite particles causes a slow but steady rearrangement of the structure taking the system to a progressively lower energy state. This concept can be understood by representing the energetic interaction of individual Laponite particles with its neighbors by a potential energy well. (Please refer to Figure S1 of Supporting Information.) In the out-of-equilibrium state, the individual particle is only occasionally able to jump out of the energy well due to limited thermal energy associated with the same. In addition, the particle is free to undergo microscopic motion while remaining surrounded by the same neighbors. ${ }^{34}$ Overall, the structural rearrangement prefers those configurations that decrease the energy of the particle as a function of time. The elastic modulus of the system, in such a case, can then be obtained as suggested by Jones. ${ }^{52}$ If we represent interparticle interactions by Hookean (quadratic) springs with the spring constant $k$, and if $b$ is the average interparticle distance, then the elastic modulus is given by $k / b$. The spring constant $k$ is equal to the second derivative of the interaction potential computed at the point of minimum energy in the well. ${ }^{52}$ By using simple scaling arguments, Jones ${ }^{52}$ suggested that $k \approx E / b^{2}$. If we assume that all the particles in the system are trapped in wells with the same energy barrier, the elastic modulus can be represented by

$$
G^{\prime}=\beta E / b^{3}
$$

where $\beta$ is a constant of proportionality. This concept can also be understood from the schematic of the energy well shown in Figure S1 of Supporting Information. The displacement of the particle by the distance $r$ enhances the energy of the particle by $(1 / 2) k r^{2}$. In the aging process, the well depth goes on increasing as a function of time; however, the average interparticle distance $b$ can be expected to remain unaffected. Consequently, in order to have the same deformation, greater energy is necessary $\left((1 / 2) k b^{2} \approx E\right)$, which again leads to $G^{\prime}=$ $\beta E / b^{3}$. Therefore, a simple picture of aging, wherein the energy well depth increases as a function of time, predicts enhancement of the elastic modulus as described in Figure S1 of Supporting Information.

Knowledge of the depth of the energy well in which particles are trapped also leads to the characteristic relaxation time through an Arrhenius relationship. ${ }^{36}$ Interestingly, relaxation time is observed to show a power law dependence on aging time in the jammed state. ${ }^{28}$ Equivalency of both equations leads to

$$
\tau=\tau_{\mathrm{m}} \exp \left(E / k_{\mathrm{B}} T\right)=A \tau_{\mathrm{m}}\left(t_{\mathrm{w}} / \tau_{\mathrm{m}}\right)^{\mu}
$$

Equation 7 models the relaxation time as cage diffusion time. In the soft glassy rheology framework, cage diffusion is considered an activated process. ${ }^{53}$ Fielding et al. ${ }^{36}$ describe the time scale $\tau_{\mathrm{m}}$ as the microscopic attempt time for the activation process. This time scale sets the rate at which material ages. Combining eqs 6 and 7 leads to an expression for elastic modulus given by

$$
G^{\prime}=\beta \frac{E}{b^{3}}=\frac{\beta k_{\mathrm{B}} T}{b^{3}} \ln A+\mu \frac{\beta k_{\mathrm{B}} T}{b^{3}} \ln \left(t_{\mathrm{w}} / \tau_{\mathrm{m}}\right)
$$

Assuming Maxwell type behavior, the viscous modulus can be related to elastic modulus by $G^{\prime \prime}=G^{\prime} / \omega \tau$. With eqs 7 and 8 , viscous modulus can be written as

$$
\begin{aligned}
\ln G^{\prime \prime}= & -\ln \left(\omega A \tau_{\mathrm{m}}\right)+\ln \left[\frac{\beta k_{\mathrm{B}} T}{b^{3}} \ln A+\mu \frac{\beta k_{\mathrm{B}} T}{b^{3}}\right. \\
& \left.\ln \left(t_{\mathrm{w}} / \tau_{\mathrm{m}}\right)\right]-\mu \ln \left(t_{\mathrm{w}} / \tau_{\mathrm{m}}\right)
\end{aligned}
$$

Interestingly, eq 9 qualitatively explains the decrease in the viscous modulus as a function of aging time at long times (last term) as shown in Figure 1. It should be noted that Figure 1 shows the decrease in $G^{\prime \prime}$ is stronger for experiments carried out at shorter idle times. Equation 9 suggests that, on a double logarithmic scale, $G^{\prime \prime}$ decreases with slope $\mu$. Remarkably, the decrease in $\mu$ at greater idle times as shown in Figure 4 nicely compliments the weakening slope of $G^{\prime \prime}$ at longer idle times as observed in Figure 1.

Overall, Figure 5 discusses how the relaxation dynamics changes from a very rapid exponential enhancement on day 13 to the strong power law dependence over days 21-56 (hyperaging) to the linear dependence in the limit of very long idle times. According to our simple model description, eq 8 suggests that the energy well depth increases with respect to time as $E \propto$ $\mu k_{\mathrm{B}} T \ln \left(t_{\mathrm{w}} / \tau_{\mathrm{m}}\right)$. Owing to the greater value of $\mu$, enhancement of the energy well depth of a shear-rejuvenated Laponite suspension is very rapid over the initial duration. In the limit of very long idle times, however, the rate of deepening of the energy well becomes steady, leading to a linear relation on aging time. Usually, for glassy materials the linear dependence of relaxation time on aging time is considered to be a signature characteristic. Struik, ${ }^{28}$ based on overwhelming data on glassy polymers as well as theoretical arguments, argued that factor $\mu$ should tend to unity in the limit of long times. Remarkably, our experiments on Laponite suspensions also suggest the same phenomenon.

The qualitative discussion explaining the enhancement of the elastic modulus, the broadness of the maxima of the viscous moduli, and the behavior of $\mu$ is based on the assumption that all the particles are trapped in energy wells having the depth $E$. However, in reality there exists a distribution of well depths in which particles are arrested $\left(E_{\mathrm{i}}\right)$. Such a distribution also leads to the distribution in relaxation times through the dependence $\tau_{\mathrm{i}}=\tau_{\mathrm{m}} \exp \left(E_{\mathrm{i}} / k_{\mathrm{B}} T\right)$. Owing to the aging dynamics, the particles lower their energy with time, which causes a slowing down of the relaxation dynamics. In Figure 2, the creep curves obtained at various aging times are shown to superimpose when plotted in the effective time domain. However, such superposition is possible only if all the relaxation modes evolve with the same dependence on aging time ( $\mu$ for all the modes remains constant). ${ }^{37}$ Consequently, all the modes can be seen to be increase with aging time, keeping the shape of the spectrum constant. (Please refer to Figure S2 of Supporting Information). In Figure 3, the time-aging time superpositions obtained on various idle times are shown to have self-similar curvatures, which lead to a comprehensive superposition. In order to observe such a superposition, it is again necessary that the 
shape of the relaxation time distribution should remain unaffected as a function of idle time. The typical procedure, which is followed during the experiments, involves shear melting (mechanical quench) to be carried out on the samples. Shear melting induces a strong deformation field in the material, fluidizing the jammed entities, thereby enhancing their energy (reducing the energy well depth). Subsequent to shear melting, particles occupy the energy wells with a distribution of well depths. However, as shown in Figure 1, the shear melting carried out on greater idle times does not rejuvenate the sample to the same initial state. This may be due to a certain structural formation which is too strong to be destroyed by the induced shear (energy wells are too deep compared to strain-induced energy). Therefore, the relaxation time distribution subsequent to shear melting, on progressively higher idle times, shifts to deeper well depths, preserving its shape.

Under constant temperature and constant pressure conditions, which is the case with the present experiments, the evolution of the structure takes place in order to lower the Gibbs free energy of the system. ${ }^{54}$ (Since the suspension of Laponite is in aqueous media and ergodicity breaking occurs over a particle length-scale, the volume of suspension does not change while it ages. Consequently, the aging process in a Laponite suspension is also a constant volume-constant temperature process. Therefore, lowering of the Helmholtz free energy also characterizes the aging process. ${ }^{54}$ ) Let us assume that at any idle time the specific Gibbs free energy of the system immediately after the shear melting is stopped is $g_{0}$. On the other hand, let the lowest possible Gibbs free energy of the system (corresponding to the equilibrium state) be $g_{\infty}$. Therefore, during an aging process the Gibbs free energy of a system $(g)$ decreases from $g_{0}$ toward $g_{\infty}$ as a function of time. If the evolution of Gibbs free energy is assumed to be a first-order process, we have ${ }^{28,55}$

$$
\frac{\mathrm{d} g}{\mathrm{~d} t}=-\frac{g-g_{\infty}}{\tau(t)}
$$

The difference $\left(g-g_{\infty}\right)$ can be represented as excess Gibbs free energy. The rate of change of relaxation time can then be related to the dependence of relaxation time on excess Gibbs free energy as

$$
\frac{\mathrm{d} \tau(t)}{\mathrm{d} t}=-\frac{\mathrm{d} \ln \tau(t)}{\mathrm{d} \ln \left(g-g_{\infty}\right)}=f[g(t)]
$$

The logarithmic dependence of relaxation time on aging time can then be represented by ${ }^{28}$

$$
\mu=\frac{\mathrm{d} \ln \tau}{\mathrm{d} \ln t}=\frac{t f[g(t)]}{\int_{0}^{t} f\left[g\left(t^{\prime}\right)\right] \mathrm{d} t^{\prime}+\tau_{\mathrm{b}}}
$$

where $\tau_{\mathrm{b}}$ is relaxation time at the beginning of aging $(t=0$ and $\left.g=g_{0}\right)$. Equation 12 can be easily rearranged to give

$$
\frac{1}{\mu}=\int_{0}^{1} \varphi \mathrm{d} \zeta+\frac{\tau_{\mathrm{b}}}{t f[g(t)]}
$$

where $\varphi\left(t^{\prime}, t\right)=f\left[g\left(t^{\prime}\right)\right] / f[g(t)]$ and $\zeta=t^{\prime} / t$. Therefore, as $\zeta$ approaches unity $\left(t^{\prime} \rightarrow t\right), \varphi\left(t^{\prime}, t\right)$ also approaches unity $(\varphi \rightarrow$ $1)$. The second term on the right-hand side of eq 13 is expected to become negligible in the limit of large aging times. Therefore, in order to observe hyper-aging $(\mu>1), \varphi$ must be an increasing function of $\zeta(\mathrm{d} \varphi / \mathrm{d} \zeta>1)$. On the other hand, in order to observe a linear dependence of relaxation time on aging time $(\mu \approx 1), \varphi$ must remain constant over the greater duration of $\zeta(\mathrm{d} \varphi / \mathrm{d} \zeta \approx 0)$. As shown in Figure 5, we observe relaxation time to follow a power law dependence on aging time given by $\tau=A \tau_{\mathrm{m}}^{1-\mu} t^{\mu}$, which leads to

$$
\varphi=\zeta^{\mu-1}
$$

In Figure 6a, we have plotted eq 14 describing the behavior of $\varphi$ with respect to $\zeta$ for various values of $\mu$. In addition, the

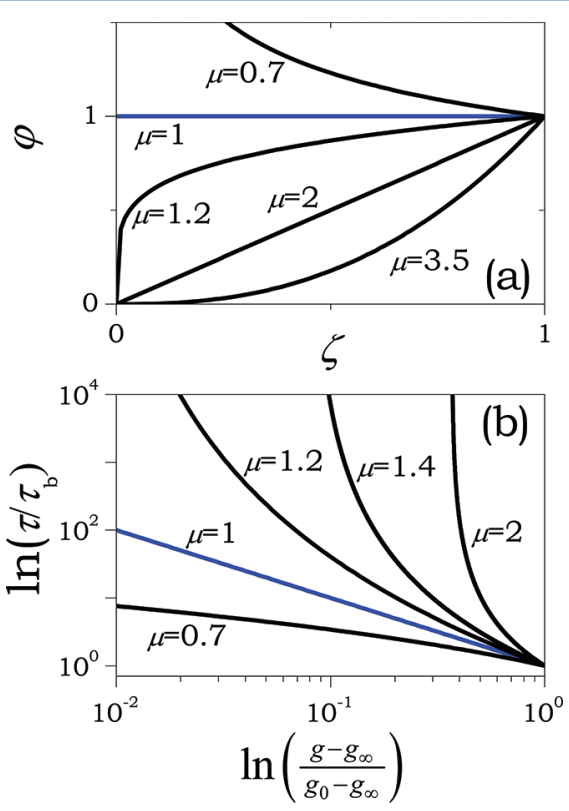

Figure 6. Schematic representing (a) dependence of $\varphi$ on dimensionless time $\zeta$ (eq 14) and (b) dependence of relaxation time on excess Gibbs free energy (eq 15 with $A=1$ and $\tau_{\mathrm{m}}=\tau_{\mathrm{b}}$ ).

dependence of relaxation time on excess Gibbs free energy for $\tau$ $=A \tau_{\mathrm{m}}^{1-\mu} t^{\mu}$ can be obtained from eq 11 as

$$
\begin{array}{ll}
\frac{\tau_{\mathrm{b}}^{\nu}}{\tau^{\nu}}=1+(\mu-1) A^{1 / \mu}\left(\frac{\tau_{\mathrm{b}}}{\tau_{\mathrm{m}}}\right)^{\nu} \ln \left(\frac{g-g_{\infty}}{g_{0}-g_{\infty}}\right) \mu>1 \\
\frac{\tau}{\tau_{\mathrm{b}}}=\left(\frac{g-g_{\infty}}{g_{0}-g_{\infty}}\right)^{-A} & \mu=1
\end{array}
$$

where $\nu=(\mu-1) / \mu$. For values of $\mu>1$, relaxation time diverges at $\ln \left[\left(g-g_{\infty}\right) /\left(g_{0}-g_{\infty}\right)\right]=\left[A^{-1 / \mu}\left(\tau_{\mathrm{m}} / \tau_{\mathrm{b}}\right)^{\nu} /(1-\mu)\right]$ leading to cessation of aging. In Figure $6 \mathrm{~b}$, we have plotted eq 15 for various values of $\mu$ by considering a special case of $A=1$ and $\tau_{\mathrm{b}}=\tau_{\mathrm{m}}$. Equation 15 and its description in Figure $6 \mathrm{~b}$ clearly suggests that the system can never approach an equilibrium state $\left(g=g_{\infty}\right)$, with $\mu>1$. (Interestingly, the limit $\mu \gg 1$ can be considered as a granular limit, as the microscopic rearrangement is frozen in granular media and the system remains in a disordered higher-energy state indefinitely.) On the other hand, for $\mu \leq 1$, aging continues and the equilibrium state is approached in the limit of a very large time $(t \rightarrow \infty)$. Our observation, described in Figure 4 suggests that the dependence of relaxation time on excess Gibbs free energy changes from strongly diverging dependence $(\mu>1)$ at shorter idle times to a linear dependence at longer idle times. Therefore, by slowly transforming the relaxation dynamics from a hyper-aging 
regime to linear regime, continuing aging dynamics is facilitated in an aqueous Laponite suspension.

\section{CONCLUSION}

In this work, we study the long-term relaxation dynamics of an aqueous suspension of nanoclay Laponite through the effective time theory. Oscillatory and creep experiments were performed on shear-rejuvenated suspension samples up to 180 days after preparation. Typically, the elastic modulus of the suspension shows enhancement as a function of time. Viscous modulus, which increases at the beginning of the aging process, shows a decrease after crossing over the elastic modulus. Experiments performed on later dates since the preparation of the suspension (idle time) cause evolution of $G^{\prime}$ to shift to a lower time scale while broadening the maxima for $G^{\prime \prime}$. Owing to the enhanced relaxation time of the suspension, creep experiments induce lesser strain in the sample for experiments carried out at greater aging times, thereby invalidating the Boltzmann superposition principle. In order to apply this principle, we transform the real time to effective time by rescaling the time-dependent relaxation processes by a constant relaxation time. Since the relaxation time is constant in the effective time domain, the Boltzmann superposition principle produces a superposition of all the creep data. Superposition also leads to a relationship between relaxation time and aging time, which typically has a power law-type dependence in the glassy domain $\left(G^{\prime}>G^{\prime \prime}\right)$. We observe that the power law exponent $\mu$, which suggests logarithmic dependence of relaxation time on aging time, is significantly greater than unity (hyper-aging behavior) at small idle times. The value of $\mu$ sharply decreases with idle time, and in the limit of large idle times, a linear dependence of relaxation time on aging time is observed $(\mu \approx 1)$. The consideration of physical aging as a firstorder process suggests that, for hyper-aging dynamics $(\mu>1)$, relaxation time diverges, terminating the process of aging over finite time scales. Therefore, in order to have aging continue in the system so as to eventually get closer to an equilibrium state, $\mu$ must approach unity. We indeed observe $\mu$ to approach unity at very large idle times. Interestingly, the observation of linear dependence in the limit of very long times is akin to that observed for glassy polymeric materials.

Even though the dependence of relaxation time on aging time changes for experiments carried out at greater idle times, superpositions obtained on various idle times show self-similar curvature. We obtain a comprehensive superposition by horizontally shifting the individual superpositions obtained on various idle times. The existence of such a comprehensive superposition suggests that the shape of the relaxation time distribution remains unaffected during the aging and rejuvenation process, though the average value of relaxation time may undergo a change. We also propose a simple model that qualitatively explains various the rheological observations very well.

\section{ASSOCIATED CONTENT}

\section{S Supporting Information}

Schematic representation of a particle trapped in an energy landscape and the effect of shear melting at different idle times on energy well distribution. This material is available free of charge via the Internet at http://pubs.acs.org.

\section{AUTHOR INFORMATION}

\section{Corresponding Author}

*E-mail: joshi@iitk.ac.in.

\section{Notes}

The authors declare no competing financial interest.

\section{ACKNOWLEDGMENTS}

Financial support from Department of Science Technology, Government of India through IRHPA scheme is greatly acknowledged.

\section{REFERENCES}

(1) Meunier, A. Clays; Springer: Berlin, 2005.

(2) Van Olphen, H. An Introduction to Clay Colloid Chemistry; Wiley: New York, 1977.

(3) Ruzicka, B.; Zaccarelli, E.; Zulian, L.; Angelini, R.; Sztucki, M.; Moussaïd, A.; Narayanan, T.; Sciortino, F. Observation of empty liquids and equilibrium gels in a colloidal clay. Nat. Mater. 2011, 10, $56-60$.

(4) Ruzicka, B.; Zulian, L.; Zaccarelli, E.; Angelini, R.; Sztucki, M.; Moussaid, A.; Ruocco, G. Competing Interactions in Arrested States of Colloidal Clays. Phys. Rev. Lett. 2010, 104, 085701.

(5) Joshi, Y. M. Model for cage formation in colloidal suspension of laponite. J. Chem. Phys. 2007, 127, 081102.

(6) Shalkevich, A.; Stradner, A.; Bhat, S. K.; Muller, F.; Schurtenberger, P. Cluster, Glass, and Gel Formation and Viscoelastic Phase Separation in Aqueous Clay Suspensions. Langmuir 2007, 23, $3570-3580$.

(7) Cummins, H. Z. Liquid, glass, gel: The phases of colloidal Laponite. J. Non-Cryst. Solids 2007, 353, 3891-3905.

(8) Bonn, D.; Kellay, H.; Tanaka, H.; Wegdam, G.; Meunier, J. Laponite: What is the difference between a gel and a glass. Langmuir 1999, 15, 7534-7536.

(9) Joshi, Y. M.; Reddy, G. R. K.; Kulkarni, A. L.; Kumar, N.; Chhabra, R. P. Rheological Behavior of Aqueous Suspensions of Laponite: New Insights into the Ageing Phenomena. Proc. R. Soc. London, Ser. A 2008, 464, 469-489.

(10) Gupta, R.; Baldewa, B.; Joshi, Y. M., Time Temperature Superposition in Soft Glassy Materials. Soft Matter 2012; DOI:10.1039/C2SM07071E. .

(11) http://www.laponite.com.

(12) Kroon, M.; Vos, W. L.; Wegdam, G. H. Structure and formation of a gel of colloidal disks. Phys. Rev. E 1998, 57, 1962-1970.

(13) Shahin, A.; Joshi, Y. M.; Ramakrishna, S. A. Interface-Induced Anisotropy and the Nematic Glass/Gel State in Jammed Aqueous Laponite Suspensions. Langmuir 2011, 27, 14045-14052.

(14) Tawari, S. L.; Koch, D. L.; Cohen, C. Electrical double-layer effects on the Brownian diffusivity and aggregation rate of Laponite clay particles. J. Colloid Interface Sci. 2001, 240, 54-66.

(15) Shahin, A.; Joshi, Y. M. Irreversible Aging Dynamics and Generic Phase Behavior of Aqueous Suspensions of Laponite. Langmuir 2010, 26, 4219-4225.

(16) Ruzicka, B.; Zaccarelli, E. A fresh look at Laponite phase diagram. Soft Matter 2011, 7, 1268-1286.

(17) Jabbari-Farouji, S.; Wegdam, G. H.; Bonn, D. Gels and Glasses in a Single System: Evidence for an Intricate Free-Energy Landscape of Glassy Materials. Phys. Rev. Lett. 2007, 99, 065701-4.

(18) Ruzicka, B.; Zulian, L.; Angelini, R.; Sztucki, M.; Moussaid, A.; Ruocco, G. Arrested state of clay-water suspensions: Gel or glass? Phys. Rev. E 2008, 77, 020402-4.

(19) Mourchid, A.; Delville, A.; Lambard, J.; Lecolier, E.; Levitz, P. Phase diagram of colloidal dispersions of anisotropic charged particles: Equilibrium properties, structure, and rheology of laponite suspensions. Langmuir 1995, 11, 1942-1950.

(20) Mongondry, P.; Tassin, J. F.; Nicolai, T. Revised state diagram of Laponite dispersions. J. Colloid Interface Sci. 2005, 283, 397-405. 
(21) Sun, K.; Kumar, R.; Falvey, D. E.; Raghavan, S. R. Photogelling Colloidal Dispersions Based on Light-Activated Assembly of Nanoparticles. J. Am. Chem. Soc. 2009, 131, 7135-7141.

(22) Dijkstra, M.; Hansen, J.-P.; Madden, P. A. Statistical model for the structure and gelation of smectite clay suspensions. Phys. Rev. E 1997, 55, 3044-3053.

(23) Jabbari-Farouji, S.; Tanaka, H.; Wegdam, G. H.; Bonn, D. Multiple nonergodic disordered states in Laponite suspensions: A phase diagram. Phys. Rev. E 2008, 78, 061405-10.

(24) Gabriel, J.-C. P.; Sanchez, C.; Davidson, P. Observation of nematic liquid-crystal textures in aqueous gels of smectite clays. J. Phys. Chem. 1996, 100, 11139-11143.

(25) Lemaire, B. J.; Panine, P.; Gabriel, J. C. P.; Davidson, P. The measurement by SAXS of the nematic order parameter of laponite gels. Europhys. Lett. 2002, 59, 55-61.

(26) Michot, L. J.; Bihannic, I.; Maddi, S.; Funari, S. S.; Baravian, C.; Levitz, P.; Davidson, P. Liquid-crystalline aqueous clay suspensions. Proc. Natl. Acad. Sci. U.S.A. 2006, 103, 16101-16104.

(27) Willenbacher, N. Unusual thixotropic properties of aqueous dispersions of Laponite RD. J. Colloid Interface Sci. 1996, 182, 501510.

(28) Struik, L. C. E. Physical Aging in Amorphous Polymers and Other Materials; Elsevier: Houston, 1978.

(29) Ovarlez, G.; Coussot, P. Physical age of soft-jammed systems. Phys. Rev. E 2007, 76, 011406.

(30) Negi, A. S.; Osuji, C. O. Time-resolved viscoelastic properties during structural arrest and aging of a colloidal glass. Phys. Rev. E 82, 031404.

(31) Sibani, P.; Kenning, G. G. Origin of end-of-aging and subaging scaling behavior in glassy dynamics. Phys. Rev. E 2010, 81, 011108.

(32) Rinn, B.; Maass, P.; Bouchaud, J.-P. Hopping in the glass configuration space: Subaging and generalized scaling laws. Phys. Rev. B 2001, 64, 104417.

(33) Bissig, H.; Romer, S.; Cipelletti, L.; Trappe, V.; Schurtenberger, P. Intermittent dynamics and hyper-aging in dense colloidal gels. PhysChemComm 2003, 6, 21-23.

(34) Bandyopadhyay, R.; Liang, D.; Yardimci, H.; Sessoms, D. A.; Borthwick, M. A.; Mochrie, S. G. J.; Harden, J. L.; Leheny, R. L. Evolution of particle-scale dynamics in an aging clay suspension. Phys. Rev. Lett. 2004, 93.

(35) Shahin, A.; Joshi, Y. M. Prediction of long and short time rheological behavior in soft glassy materials. Phys. Rev. Lett. 2011, 106, 038302.

(36) Fielding, S. M.; Sollich, P.; Cates, M. E. Aging and rheology in soft materials. J. Rheol. 2000, 44, 323-369.

(37) Baldewa, B.; Joshi, Y. M. Delayed Yielding in Creep, Time Stress Superposition and Effective Time Theory for a soft Glass. Soft Matter 2012, 8, 789-796.

(38) Mongondry, P.; Nicolai, T.; Tassin, J.-F. Influence of pyrophosphate or polyethylene oxide on the aggregation and gelation of aqueous laponite dispersions. J. Colloid Interface Sci. 2004, 275, 191-196.

(39) Zebrowski, J.; Prasad, V.; Zhang, W.; Walker, L. M.; Weitz, D. A. Shake-gels: shear-induced gelation of laponite-PEO mixtures. Colloids Surf., A 2003, 213, 189-197.

(40) Bird, R. B.; Armstrong, R. C.; Hassager, O. Dynamics of Polymeric Liquids, Fluid Mechanics; Wiley-Interscience: New York, 1987.

(41) Schosseler, F.; Kaloun, S.; Skouri, M.; Munch, J. P. Diagram of the aging dynamics in laponite suspensions at low ionic strength. Phys. Rev. E 2006, 73, 021401.

(42) Kaloun, S.; Skouri, R.; Skouri, M.; Munch, J. P.; Schosseler, F. Successive exponential and full aging regimes evidenced by tracer diffusion in a colloidal glass. Phys. Rev. E 2005, 72, 011403.

(43) Bellour, M.; Knaebel, A.; Harden, J. L.; Lequeux, F.; Munch, J.P. Aging processes and scale dependence in soft glassy colloidal suspensions. Phys. Rev. E 2003, 67, 031405.
(44) Awasthi, V.; Joshi, Y. M. Effect of temperature on aging and time-temperature superposition in nonergodic laponite suspensions. Soft Matter 2009, 5, 4991-4996.

(45) Joshi, Y. M.; Reddy, G. R. K. Aging in a colloidal glass in creep flow: Time-stress superposition. Phys. Rev. E 2008, 77, 021501-4.

(46) Cloitre, M.; Borrega, R.; Leibler, L. Rheological aging and rejuvenation in microgel pastes. Phys. Rev. Lett. 2000, 85, 4819-4822.

(47) Cipelletti, L.; Ramos, L.; Manley, S.; Pitard, E.; Weitz, D. A.; Pashkovski, E. E.; Johansson, M. Universal non-diffusive slow dynamics in aging soft matter. Faraday Discuss. 2003, 123, 237-251.

(48) Agarwal, P.; Qi, H.; Archer, L. A. The Ages in a Self-Suspended Nanoparticle Liquid. Nano Lett. 2009, 10, 111-115.

(49) Sibani, P.; Hoffmann, K. H. Hierarchical-models for aging and relaxation of spin-glasses. Phys. Rev. Lett. 1989, 63, 2853-2856.

(50) Hodge, I. M. Physical aging in polymer glasses. Science 1995, 267, 1945-1947.

(51) Guo, Y.; Zhang, C.; Lai, C.; Priestley, R. D.; D’Acunzi, M.; Fytas, G. Structural Relaxation of Polymer Nanospheres under Soft and Hard Confinement: Isobaric versus Isochoric Conditions. ACS Nano 2011, 5, 5365-5373.

(52) Jones, R. A. L. Soft Condensed Matter; Oxford University Press: Oxford, 2002.

(53) Sollich, P.; Lequeux, F.; Hebraud, P.; Cates, M. E. Rheology of soft glassy materials. Phys. Rev. Lett. 1997, 78, 2020-2023.

(54) Callen, H. B. Thermodynamics and an introduction to thermostatistics; John Wiley \& Sons: New York, 1985; p 13.

(55) Shaw, M. T.; MacKnight, W. J. Introduction to Polymer Viscoelasticity, 3rd ed.; Wiley: New York, 2005. 


\title{
SUPPORTING INFORMATION
}

\section{Hyper-aging Dynamics of Nano-clay Suspension}

\author{
A.Shahin and Yogesh M Joshi* \\ Department of Chemical Engineering, Indian Institute of Technology Kanpur, \\ Kanpur 208016. INDIA. \\ *E-Mail: joshi@iitk.ac.in
}



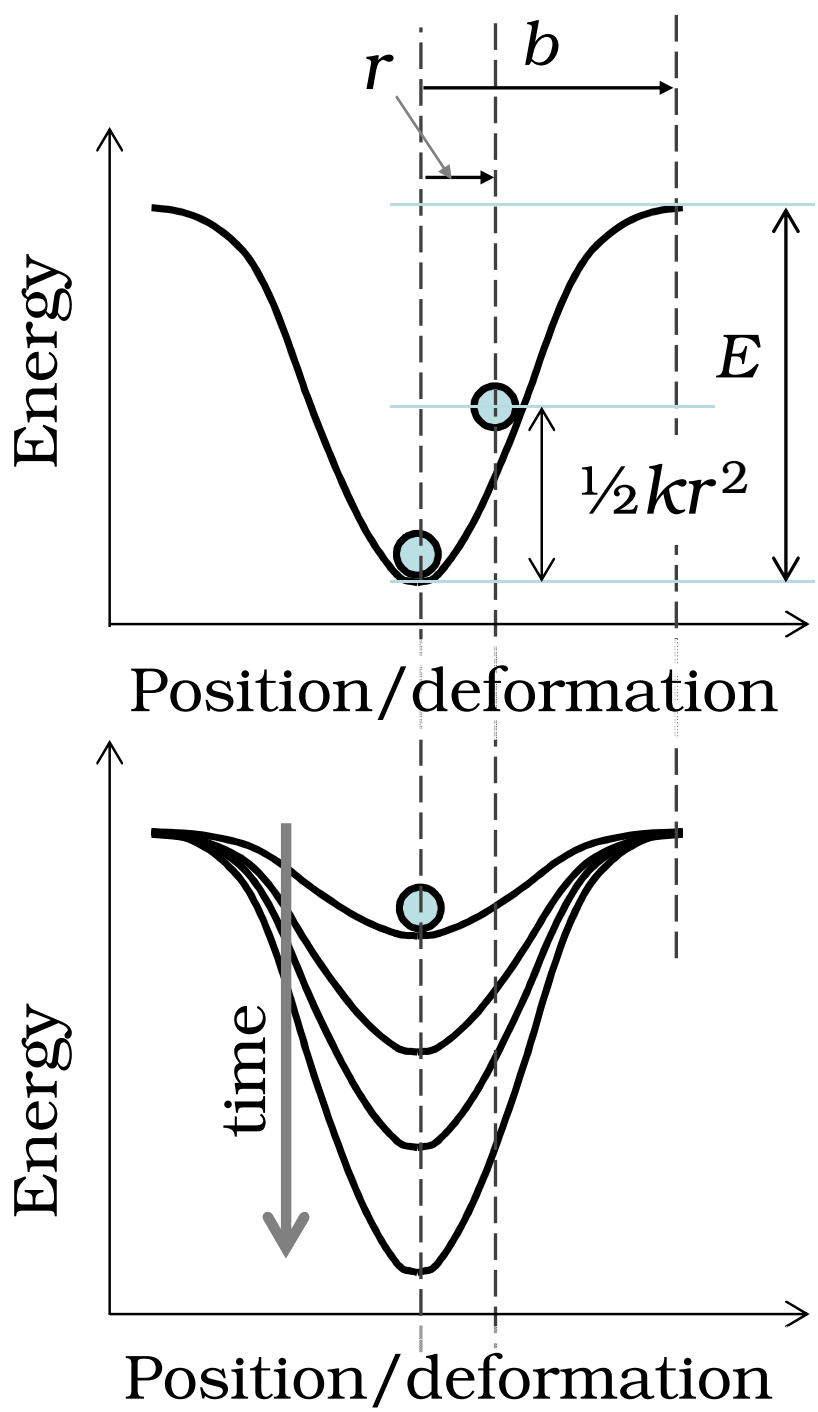

Figure S1. Schematic representing particle trapped in energy well under a deformation field. Bottom schematic describes aging of a well in which well depth goes on increasing as a function of time. 


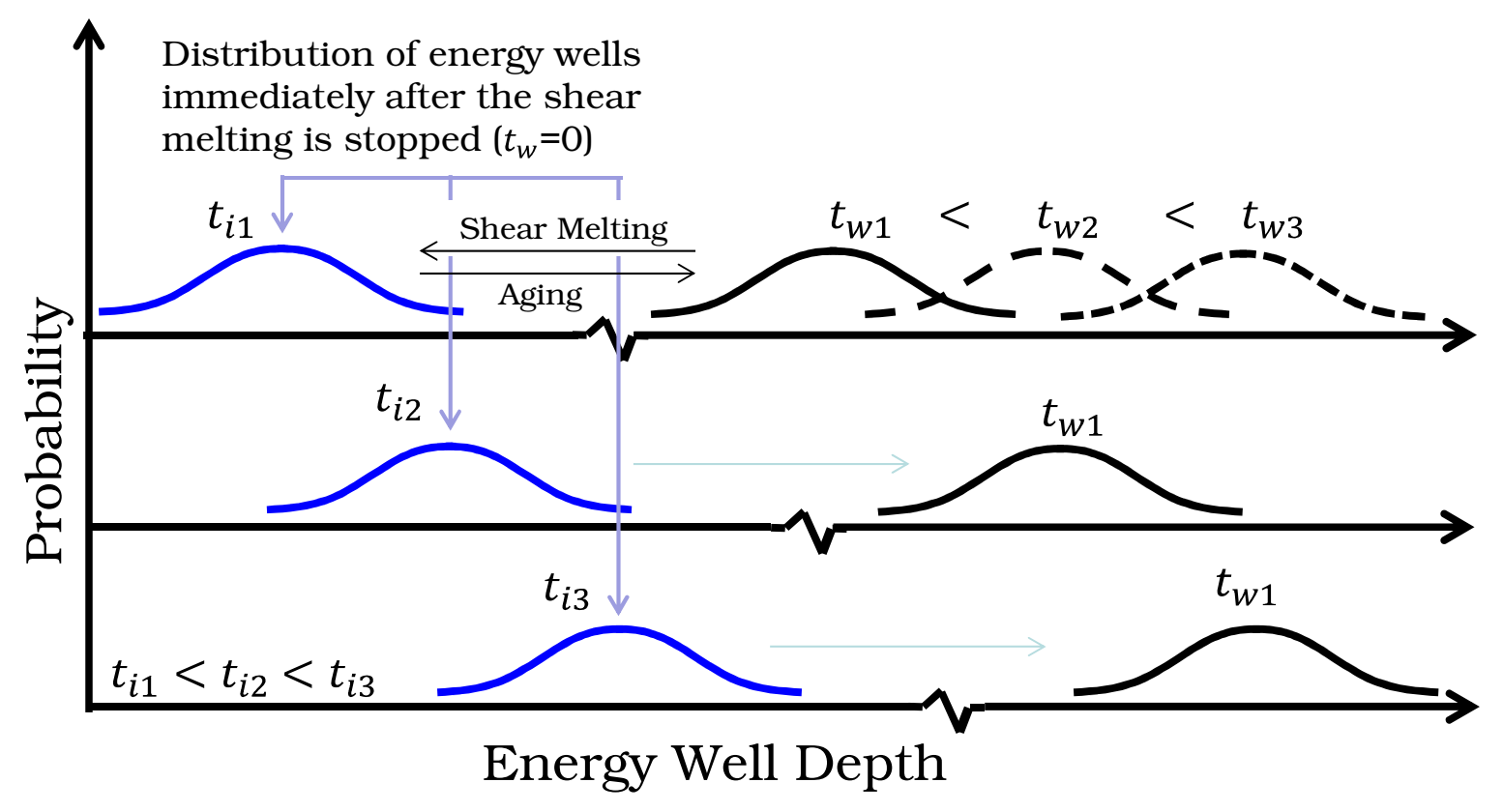

Figure S2. Effect of shear melting at different idle times on energy well distribution. Owing to irreversible aging, shear melting on greater idle times shifts the energy well distribution dome to higher well depths. Consequently suspension has greater average relaxation time for experiments carried out at same aging times but greater idle times. Self-similarity of the shape of the distribution dome as a function of aging time and idle time is a necessary condition to observe superpositions shown in figure 3. 